\title{
LA EVALUACIÓN DE COMPETENCIAS EN LAS CIENCIAS SOCIALES DE LA EDUCACIÓN PRIMARIA
}

The evaluation of competences in the Social Sciences of Primary Education A avaliação de competências em Ciências Sociais da Educação Primária

\author{
Alejandro Bermúdez Medel \\ Universidad de Valladolid. Teléfono 636785571. Correo electrónico: \\ bermedel@telefonica.net
}

\begin{abstract}
Resumen
El currículo formal de la Educación Primaria plantea, pero no desarrolla eficazmente, un modelo de evaluación por competencias. Sin embargo, una importante parte de los maestros de la etapa no lo consideran en su totalidad o simplemente no lo aplican debido a diversas causas (formación, tiempo...). El proyecto piloto "Conozco y reconozco mi entorno” (Segovia, España), propone un sistema de evaluación, basado en el cuadro de control, en el marco de un proceso de integración del entorno local (microespacio) y los contenidos del currículo formal (macroespacio) y de la aplicación del primero como vehículo-instrumento en el proceso enseñanza-aprendizaje.

Palabras clave: Evaluación por competencias; Educación Primaria; Ciencias Sociales
\end{abstract}

\begin{abstract}
The Primary Education formal curricula proposes, although not effectively develops, an appraisal model based on capacities. Notwithstanding this, an important part of the teachers of that stage do not consider it completely, or they just do not apply it because of diverse reasons (formation, schedule,...). The mock-up project "I know and recognize my environment" (Segovia, Spain) proposes an appraisal system based on the control dashboard, which is within the local environment (microspace) integration process. Furthermore, it is alto based on the contents of the formal curricula (macrospace) and the application of the local environment as a vehicle/instrument in the teaching/learning process.
\end{abstract}

Keywords: Competency assessment; Primary Education; Social Sciences 


\section{Resumo}

O currículo formal da Educação Primária apresenta, mas não efetivamente, um modelo de avaliaçao baseado em competências. Porém, uma parte importante dos professores deste ciclo de ensino não o considera na sua totalidade ou simplesmente não se aplicam devido a várias causas (formação, tempo,...). O projecto-piloto “Eu conheço e reconheço o meu ambiente” (Segovia, Espanha) propõe um sistema de avaliação, com base no quadro de controle, no âmbito de um processo de integração do ambiente local (microespaço). Considera-se também o conteúdo do currículo formal (macroespaço) e a aplicação do ambiente local como veículo-instrumento no processo de ensino e aprendizagem.

Palavras-chave: Avaliação por competências; Educação Primária; Ciências Sociais

\section{Introducción}

El currículo formal (Real Decreto 126/2014; Orden ECD/65/2015; en Castilla y León, Decreto 26/2016) plantea un modelo de desarrollo y evaluación competencial en el proceso enseñanza-aprendizaje de la Educación Primaria. Sin embargo, de acuerdo con nuestra experiencia en centros educativos de diversa tipología, localización geográfica y características socioeconómicas del entorno, la implementación en las aulas es, en la realidad, muy limitada. Los centros educativos han abordado este complejo asunto con diversos enfoques y, por su parte, las editoriales de material educativo, con distinto grado de éxito, han aportado su colaboración con el objeto de facilitar al maestro el proceso.

El proyecto “Conozco y reconozco mi entorno” (Segovia, España. 2016) plantea un procedimiento didáctico basado en el medio local como vehículo del proceso enseñanza aprendizaje y sigue un método inductivo-deductivo que opera desde los contenidos facilitados por el microespacio inmediato a los propios del macroespacio generalista. En el marco de este proyecto, se ha desarrollado un sistema de evaluación que incluye instrumentos (observación directa del docente, ejercicios de clase/cuaderno, salidas a la pizarra y exposiciones orales, trabajos de investigación, individuales o en equipo, pruebas escritas...) y un procedimiento de calificación centralizado en un 
cuadro de control o sistema de criterios, indicadores y estándares (rúbrica). Esta propuesta se halla en la actualidad en fase de aplicación.

Desde la Teoría de la Educación (véase fundamentación teórica del currículo formal: Orden ECD/65/2015, Recomendación 2006/962 EC y DeSeCo 2003), las competencias deben medirse en grandes tramos (por etapas), ya que es difícil, incluso inadecuado, acotarlas por ciclos o niveles y mucho menos por cursos. Pese a ello, no pueden obviarse totalmente en éste último estadio de la escala. Por eso, recurrimos a los objetivos de etapa, curso o área. Éstos no dejan de ser concreciones de las competencias. Para llegar a éstas últimas, el currículo formal establece contenidos, criterios de evaluación y estándares de aprendizaje e introduce el concepto de "perfil de competencia”, entendido como el conjunto de estándares, de la suma de áreas, que se refieren a cada una de ellas. El problema es que, esta relación (estándares-competencia) no se materializa directamente en ninguna normativa (ni siquiera en ECD/65/2015 donde se define el concepto), dejando en manos de los maestros esta compleja tarea cuando proceden a efectuar la programación didáctica.

Por otro lado, aunque las escasas referencias en el currículo formal a los indicadores parecen presentarlos como instrumentos de registro (rúbricas o escalas de evaluación) y no tanto como evidencias o síntomas de logro o cumplimiento, la realidad es que en los sistemas de criterios, indicadores y estándares al uso, los segundos (indicadores) representan ineludiblemente el eslabón entre los dos extremos de la cadena (criterios y estándares). El currículo, sin embargo, “salta” del criterio al estándar. Esto es una simplificación de un proceso complejo: mi objetivo es la competencia X. Imparto unos contenidos. ¿Qué debo medir? Unos criterios. ¿Cómo debo medirlos? A través de indicadores (de cumplimiento). ¿Con qué módulo de medida debo hacerlo? Con un baremo. ¿Qué nivel debo alcanzar? El estándar-impuesto (lo más concreto posible). La suma de estándares determinará el grado de consecución de la competencia.

En caso de ser necesario, y dada la ambigüedad, el indicador podría considerarse el/los resultados (síntomas, evidencias) de la aplicación del instrumento de evaluación.

\section{Método}

Nos referimos al procedimiento empleado en la fase proyectual, ya que la aplicación en el aula dispone de su propio método: 
1.- análisis de procesos en evaluación por competencias, bibliográfico o documentalmente avalados, en el marco de la actual Teoría de la Educación.

2.- análisis del currículo formal de Educación Primaria y especialmente del correspondiente a las Ciencias Sociales de cuarto curso.

3.- análisis del centro educativo piloto y su entorno: caracterización e identificación de fortalezas.

4.- análisis de la programación de aula del centro piloto.

5.- “reprogramación”: una nueva propuesta curricular que aplica el método inductivo y utiliza el entorno local como instrumento-vehículo en el proceso enseñanza-aprendizaje.

\section{Resultados y discusión}

Recogemos a continuación, a modo de ejemplo, el registro de contenidos, criterios de evaluación, estándares de aprendizaje y competencias afectadas, correspondiente a una unidad didáctica que pertenece a la programación del proyecto "Conozco y reconozco mi entorno”, aplicada de forma experimental al área de Ciencias Sociales de $4^{\circ}$ curso de Primaria en el CRA. "Los Almendros” de La Lastrilla en Segovia, 2017 en adelante (Anexo 1). El procedimiento y registro de calificaciones y valoraciones correspondiente a la unidad anterior queda recogida en el siguiente cuadro de control (Anexo 2)

\section{Conclusiones}

Se trata de una propuesta en fase de experimentación, pero se espera:

1.- una racionalización, sistematización y objetivación básica de los procesos de evaluación.

2.- un avance en la consideración de la competencia como meta a alcanzar del proceso enseñanza-aprendizaje.

3.- una relación directa y con trazabilidad entre contenidos, criterios de evaluación, estándares de aprendizaje y competencias (a través del perfil de competencia).

\section{Referencias}

Decreto 26/2016, de 21 de julio, por el que se establece el currículo y se regula la implantación, evaluación y desarrollo de la Educación Primaria en Castilla y León (BOCyL 25 de julio de 2016). 
DeSeCo, 2003. Definición y selección de competencias. Organización para la Cooperación y el desarrollo económico.

Orden ECD/65/2015, de 21 de enero, por la que se describen las relaciones entre competencias, los contenidos y los criterios de evaluación de la educación primaria, la educación secundaria obligatoria y el bachillerato.

Real Decreto 126/2014, de 28 de febrero, por el que se establece el currículo básico de la Enseñanza Primaria (BOE 52 de 1 de marzo de 2014).

Recomendación 2006/962 EC del Parlamento Europeo y del Consejo, de 18 de diciembre de 2006 sobre las competencias clave para el aprendizaje permanente. 
PROYECTO CONOZCO Y RECONOZCO

Unidad didáctica 5: "Nos movemos y nos comunicamos"

\begin{tabular}{|c|c|c|c|c|c|c|c|c|c|c|}
\hline & CONTTNOOS & CRITERIOS & FSTANDAPEC & & & $\mathrm{CON}$ & ETEN & & & \\
\hline & & & & CL & СMCT & CD & AA & $\csc$ & SIEE & CEC \\
\hline CUR CS0.1.01 & $\begin{array}{l}\text { Iniciación al conocimiento científico y su aplicación en las } \\
\text { Ciencias Sociales. }\end{array}$ & $\begin{array}{l}\text { 1. Obtenerer infomación concreta y relevante sobre hechos o fenómenos } \\
\text { previamente delimitados, utilizando diferentes fuentes (directas e } \\
\text { indirectas) siendo capaz de analizar e interpretar la información recibida. }\end{array}$ & $\begin{array}{l}\text { 1.1. Busca, selecciona y organiza información concreta y relevante, la } \\
\text { analiza, elabora conclusiones, reflexiona acerca del proceso seguido y lo } \\
\text { comunica oralmente y/o por escrito. }\end{array}$ & $\mathrm{X}$ & & $\mathrm{X}$ & $\mathrm{X}$ & & & \\
\hline CUR CS 0.1 .02 & $\begin{array}{l}\text { Recogida de información del tema a tratar, utilizando } \\
\text { diferentes fuentes (directas e indirectas) para elaborar } \\
\text { sintesis, comentarios e informe y otros trabajas de }\end{array}$ & $\begin{array}{l}\text { 2. Utilizar las TIC para obtener información, recogiendo datos para } \\
\text { aprender, realizar exposiciones, compartir conocimientos y expresar } \\
\text { contenidos de Ciencias Sociales. }\end{array}$ & $\begin{array}{l}\text { 2.1.Utiliziz lasTIC (internet, blogs, redes sociales...) para elaborar } \\
\text { trabajos con la terminologia adecuada a los temas tratados. }\end{array}$ & & & $\mathrm{X}$ & $\mathrm{X}$ & & & \\
\hline & & & $\begin{array}{l}\text { 2.2. Analiza informaciones relacionadas con el área y maneja imágenes, } \\
\text { tablas, gráficos, mapas, esquemas, resúmenes y las TIC geográficas. }\end{array}$ & & & $\mathrm{X}$ & $\mathrm{X}$ & & & \\
\hline CUR CS0.1.03 & $\begin{array}{l}\text { Utización de las TIC para buscar y seleccionar } \\
\text { información para aprender, compartir y presentar } \\
\text { conclusiones. }\end{array}$ & $\begin{array}{l}\text { 3. Desarrollar la responsabilidad, la capacidad de esfuerzo y la } \\
\text { constancia en el estudio. }\end{array}$ & $\begin{array}{l}\text { 31. Realiza las tareas encomendadas y presenta los trabajos de manera } \\
\text { ordenada, clara y limpia. }\end{array}$ & $\mathrm{X}$ & & & $\mathrm{X}$ & & & \\
\hline & & & $\begin{array}{l}\text { 3.2. Utiliza con rigor y precisión el vocabulario adquirido para elaborar } \\
\text { trabajos con la terminologia adecuada a los temas tratados. }\end{array}$ & $\mathrm{X}$ & & & $\mathrm{X}$ & & & \\
\hline & & & $\begin{array}{l}\text { 3.3. Expone oralmente, de forma clara y ordenada, contenidos } \\
\text { relacionados con el ârea, que manifiesten la comprensión de textos }\end{array}$ & $\mathrm{X}$ & & & $\mathrm{X}$ & & & \\
\hline & & & $\begin{array}{l}\text { 3.4. Domina la ortografía al nivel definido en el área de lengua para su } \\
\text { curso. }\end{array}$ & $\mathrm{X}$ & & & $\mathrm{X}$ & & & \\
\hline CUR CS 0.1 .04 & $\begin{array}{l}\text { Utilización, interpretación y lectura de diferentes } \\
\text { lenguajes textuales, ráficos códigos numerícos, } \\
\text { cartográficosy otros, del entomo social próximo. }\end{array}$ & $\begin{array}{l}\text { 4. Realizar trabajos y presentaciones a nivel individual y grupal que, } \\
\text { utilizando diferentes técnicas, supongana la buśsqueda, memorización, } \\
\text { selección y organizacióndes textos de carácter social, geográfico o } \\
\text { histórico, mostrando habilidad para trabajar tanto individualmente como } \\
\text { de manera colaborativa dentro de un equipo. }\end{array}$ & $\begin{array}{l}\text { 4.1. Realiza trabajos y presentaciones a nivel individual y grupal que } \\
\text { suponen la bús sueda, selección y organización de textos de carácter } \\
\text { geográfico, sociale histórico. }\end{array}$ & $\mathrm{X}$ & & $\mathrm{X}$ & $\mathrm{X}$ & $\mathrm{X}$ & & \\
\hline CUR CS 0.1 .05 & $\begin{array}{l}\text { Técnicas de trabajo intelectual. Elaboración de esquemas, } \\
\text { resúmenes, memorización y estructuración de la } \\
\text { información recibida. }\end{array}$ & $\begin{array}{l}\text { 5. Valorar el trabajo en equipo, mostrando actitudes de cooperación y } \\
\text { participación responsable y adoptando un comportamiento constructivo } \\
\text { que acepte las diferencias hacia las ideas y aportaciones ajenas. }\end{array}$ & $\begin{array}{l}\text { 5.1. Utiliza estrategias para realizar trabajos de forma individual y en } \\
\text { equipo, y muestra habilidades para la resolución paćifica de conflictos. } \\
\text {. }\end{array}$ & & & & $\mathrm{X}$ & $\mathrm{X}$ & $\mathrm{X}$ & \\
\hline CUR CS 0.1.06 & $\begin{array}{l}\text { Desarrollo de estratégias para organizar, memorizary } \\
\text { recuperar la información, recogiendo las ideas principales, } \\
\text { obtenidas mediante diferentes métedos s y fuentes de } \\
\text { carácter cientifico, geográfico e históico. }\end{array}$ & \begin{tabular}{|l|} 
6. Apreciar y respetar la variedad de los diferentes grupos humanos, \\
entre otros, la etnia gitana, y valorar la importancia de una convivencia \\
pacifica y tolerante entre todos ellos sobre la base de los valores \\
demorraticos y los derechos humanos universalmente compartidos. \\
\end{tabular} & $\begin{array}{l}\text { 6.1. Valora la importancia de una convivencia pacifica y tolerante entre } \\
\text { los diferentes grupos humanos sobre la base de los valores } \\
\text { democraticos y los derechos humanos universalmente compartidos. }\end{array}$ & & & & $\mathrm{X}$ & $\mathrm{X}$ & & \\
\hline CUR CS 0.1 .07 & $\begin{array}{l}\text { Estrategias para desarrollar la responsabilidida, la } \\
\text { capacidad de esfuerzo y la constancia en el estudio. }\end{array}$ & $\begin{array}{l}\text { 7. Valorar la cooperación y el dialogo como forma de evitar y resolver } \\
\text { conflictos, fomentando la igualdad entre el hombre y la mujer y los } \\
\text { valores democráticos. }\end{array}$ & $\begin{array}{l}\text { 7.1. Participa de una manera eficaz y constructiva en la vida social y crea } \\
\text { estrategias para resolver conflictos. }\end{array}$ & & & & $\mathrm{X}$ & $\mathrm{X}$ & $\mathrm{X}$ & \\
\hline & & & $\begin{array}{l}\text { 7.2. Valora la cooperación y el dialogo como forma de evitar y resolver } \\
\text { conflictos, fomentando la igualdad entre el hombre y la mujer y los } \\
\text { valores democráticos. }\end{array}$ & & & & $\mathrm{X}$ & $\mathrm{X}$ & & \\
\hline & & & $\begin{array}{l}\text { 7.3. Identifica y utiliza los códigos de conducta y los usos generalmente } \\
\text { aceptados en las distintas sociedades y entornos (escuela, familia, } \\
\text { barrio etc.). }\end{array}$ & & & & $\mathrm{X}$ & $\mathrm{X}$ & & \\
\hline CUR CS 0.1 .08 & $\begin{array}{l}\text { Fomento de técnicas de animación a la lectura de texxos } \\
\text { de divulgación de las Ciencias Sociales, de carácter social, } \\
\text { geografíco e histórico. }\end{array}$ & $\begin{array}{l}\text { 8. Desarrollar la creatividad y el espíritu emprendedor, aumentando las } \\
\text { capacidades para aprovechar la información, las ideas y presentar } \\
\text { conclusiones innovadoras, originales y creativas }\end{array}$ & $\begin{array}{l}\text { 8.1. Muestra actitudes de confianza en sí mismo, sentido crítico, } \\
\text { iniciativa personol, curiosidad, interés, creatividad en el aprendizaje y } \\
\text { espinitu emprendedor que le hacen activo ante las circunstancias que le } \\
\text { rodean. }\end{array}$ & & & & $\mathrm{X}$ & & $\mathrm{X}$ & \\
\hline & & & \begin{tabular}{|l|} 
8.2. Manifiesta autonomía en la planificación y ejecución de acciones y \\
tareas y tiene iniciativa en la toma de decisiones.
\end{tabular} & & & & $\mathrm{X}$ & $\mathrm{X}$ & $\mathrm{X}$ & \\
\hline CUR CS 0.1 .09 & $\begin{array}{l}\text { Utilización de estratégias para potenciar la cohesión del } \\
\text { grupo y e ltrabajo cooperativo desararollando habilidades } \\
\text { sociales que favorezcan la colaboración, la igualdad entre } \\
\text { hombres y mujeres y valorando la importancia de la } \\
\text { colaboración de todos. }\end{array}$ & $\begin{array}{l}\text { 9.Desarrollar actitudes de cooperación y de trabajo en equipo, asícomo } \\
\text { el hábito de asumir nuevos roles en una sociedad en continuo cambio. }\end{array}$ & $\begin{array}{l}\text { 9.1. Desarrolla actitudes de cooperación y de trabajo en equipo, valora } \\
\text { las ideas ajenas y reacciona con intuición, apertura y flexibilidad ante } \\
\text { ellas. }\end{array}$ & & & & $\mathrm{X}$ & $\mathrm{X}$ & $\mathrm{X}$ & \\
\hline CUR CS0.1.11 & $\begin{array}{l}\text { Planificacicón y gestition de proyectos con el fin de alcanzar } \\
\text { objetivos. Iniciativa emprendedora y mecanismos del } \\
\text { intercambio comercial. }\end{array}$ & & & & & & & & & \\
\hline CUR CS 0.1.12 & $\begin{array}{l}\text { Estrategias para la resolución de conflictos, utilización de } \\
\text { las normas de convivencia y valoración de la convivencia } \\
\text { pacificic y tolerante, aceptando las diferencias de los } \\
\text { distintos grapos humanos, entre ofros, del pueblog gitano. }\end{array}$ & & & & & & & & & \\
\hline PRO.CS.2. & Comunicación y transportes. & 1. Conceptualización de la comunicacción y sus tipos. & $\begin{array}{l}\text { 1.1. Reconoce en el ternitorio todos los tipos de comunicacción posible y } \\
\text { los explica. }\end{array}$ & & $\mathrm{X}$ & $\mathrm{X}$ & $\mathrm{X}$ & & & \\
\hline PRO. CS 2.1. & La red de carreteras. & $\begin{array}{l}\text { 1. Reconocimiento visual y cartográfico de carreteras. Jerarquización y } \\
\text { caracterización. }\end{array}$ & $\begin{array}{l}\text { 1.1. Identifica visualmente en el mapa al menos } 5 \text { carreteras y describe } \\
\text { sus caracteristicas. }\end{array}$ & $\mathrm{X}$ & & $\mathrm{X}$ & $\mathrm{X}$ & & & \\
\hline & & 2. Valoración social y económica de las carreteras. & 2.1. Describe el valor social y económico de las carreteras. & $\mathrm{X}$ & & & $\mathrm{X}$ & $\mathrm{X}$ & & \\
\hline PRO. CS. 2.2 . & Caminos y cañadas. & 1-Definición y reconocimiento visual y cartográfico. & $\begin{array}{l}\text { 1.1. Identifica visualmente } 2 \text { caminos y cañadas, los situa en el mapa y } \\
\text { describe su función y características. }\end{array}$ & $\mathrm{X}$ & & $\mathrm{X}$ & $\mathrm{X}$ & & & \\
\hline & & 2. Valoración histórica y económica de los caminos y cañadas. & 2.1. Describe el valor histórico y económico de estas vías basicamente. & $\mathrm{X}$ & & $\mathrm{X}$ & $\mathrm{X}$ & & & \\
\hline PRO. CS. 2.3. & Los puertos de montaña. & $\begin{array}{l}\text { 1. Definición y reconocimiento visual y cartográfico de los puertos de } \\
\text { montaña. }\end{array}$ & 1.1. Identifica en el mapa 2 puertos de montaña y describe su uso. & $\mathrm{X}$ & & $\mathrm{X}$ & $\mathrm{X}$ & & & \\
\hline & & 2. Valoración histórica y económica de los puertos de montaña. & 2.1. Describe el valor histórico y económico basicamente. & $\mathrm{X}$ & & $\mathrm{X}$ & $\mathrm{X}$ & & & \\
\hline PRO. CS. 2.4. & Las vías férreas & $\begin{array}{l}\text { 1. Definición, valoración y reconocimiento cartográfico de las vías } \\
\text { fémreas. }\end{array}$ & 1.1. Identifica en un mapa 2 vías féreas y describe su uso. & $\mathrm{X}$ & & $\mathrm{X}$ & $\mathrm{X}$ & & & \\
\hline PRO. CS 2.5. & Las vías aéreas. & 1. Reconocimiento y valoración de las vías aéreas. & $\begin{array}{l}\text { 1.1. Explica lo que son, cita } 2 \text { recoridos y describe el valor socialy } \\
\text { económico basicamente. }\end{array}$ & $\mathrm{X}$ & $\mathrm{X}$ & $\mathrm{X}$ & $\mathrm{X}$ & & & \\
\hline PRO: CS. 2.6. & Comunicaciones telefónicas y digitales. & $\begin{array}{l}\text { 1. Reconocimiento, valoración//utilidades e identificación de algunos } \\
\text { elementos en el teritorio. }\end{array}$ & $\begin{array}{l}\text { 1.1. Identifica y situa en el mapa } 4 \text { elementos de las comunicaciones } \\
\text { audiovisuales y digitites, describe sus caracterís ticas y describe su uso } \\
\text { básico: TV, movil. }\end{array}$ & $\mathrm{X}$ & $\mathrm{X}$ & $\mathrm{X}$ & $\mathrm{X}$ & $\mathrm{X}$ & & $\mathrm{X}$ \\
\hline 4.3 .1$. & La organización politica y territorial de España.t. & nesta unidad. & & & & & & & & \\
\hline CUR. & $\begin{array}{l}\text { Educación Vial: conductas y hábitos viales correctos de } \\
\text { peatones y usuarios del transporte público o privado. }\end{array}$ & $\begin{array}{l}\text { 5. Conocer y respetar las normas de circulación, fomentar la seguridad } \\
\text { vial entodos sus aspectos e identificar mapas de las vías de municación. }\end{array}$ & $\begin{array}{l}\text { 5.1.Explica nomas básicas de circulación y las consecuencias derivadas } \\
\text { del desconocimiento o incumpliniento de las mismas. }\end{array}$ & $\mathrm{X}$ & & & $\mathrm{X}$ & $\mathrm{X}$ & & \\
\hline
\end{tabular}

Anexo 1.

Registro de contenidos, criterios, estándares y competencias de una unidad didáctica. 
UD 5: "Nos movemos y nos comunicamos"

Alumno:

Instrumentos de evaluación aplicados:

CUADRO DE CONTROL: REGISTRO DE CALIFICACIÓN

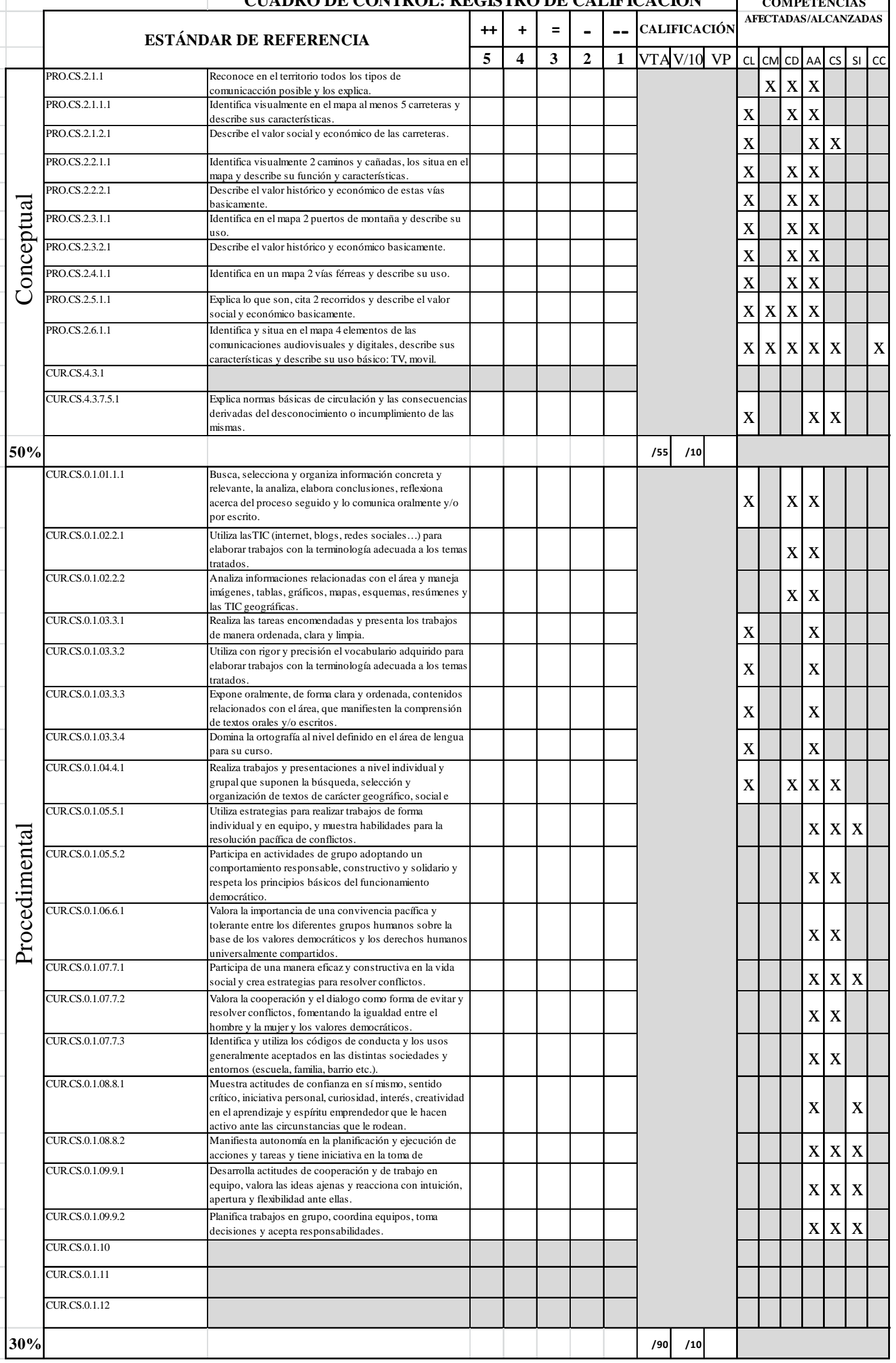

La evaluación de competencias en las Ciencias Sociales de la Educación Primaria 


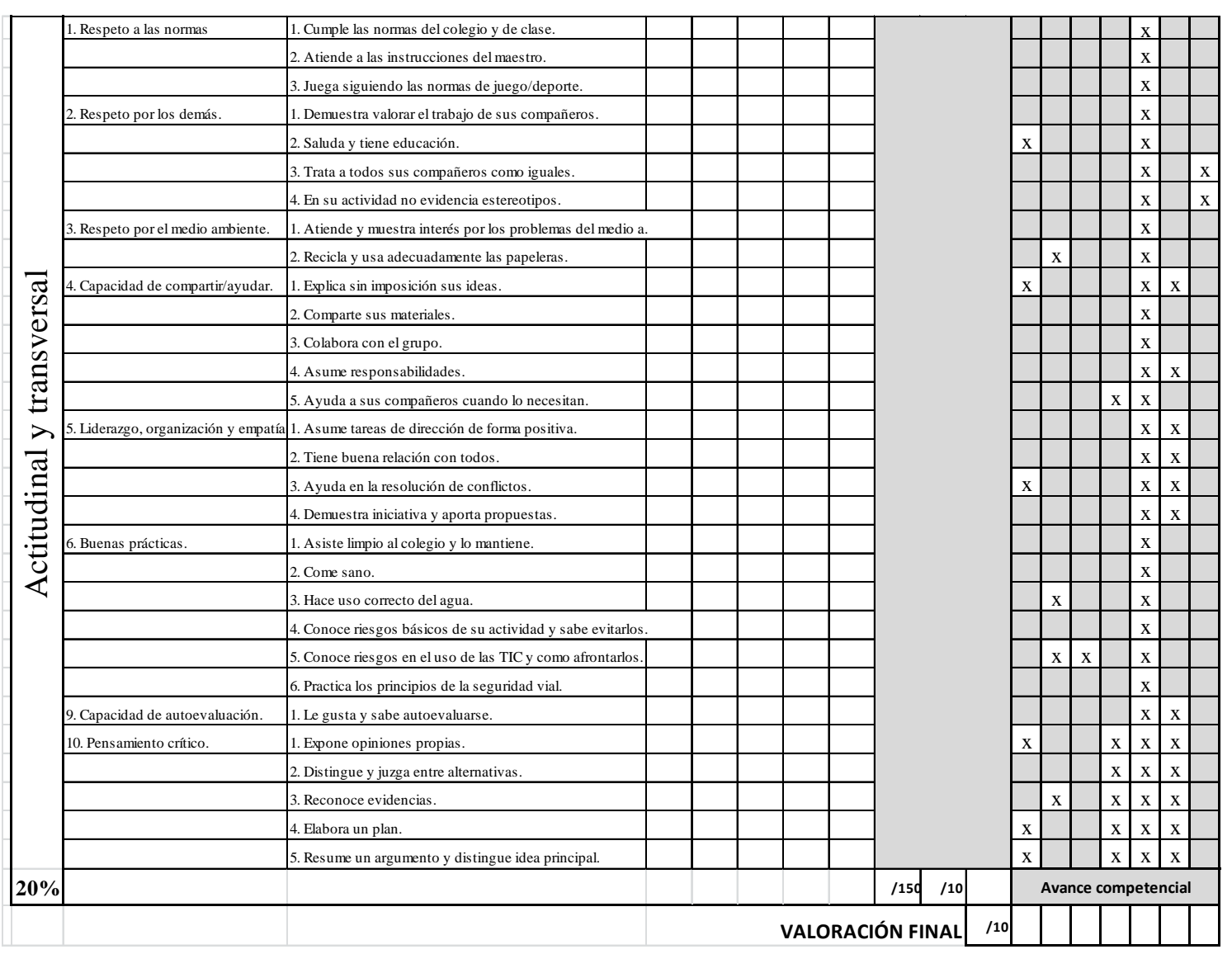

Anexo 2.

Procedimiento de calificación: cuadro de control 